\title{
Bijective mappings with generalized barycentric coordinates A counterexample
}

\section{Report}

Author(s):

Jacobson, Alec

Publication date:

2012

Permanent link:

https://doi.org/10.3929/ethz-a-007600337

Rights / license:

In Copyright - Non-Commercial Use Permitted

\section{Originally published in:}

Technical report / Departement of Computer Science, ETH Zürich 780 


\title{
Bijective mappings with generalized barycentric coordinates: a counterexample
}

\author{
Alec Jacobson ETH Zurich
}
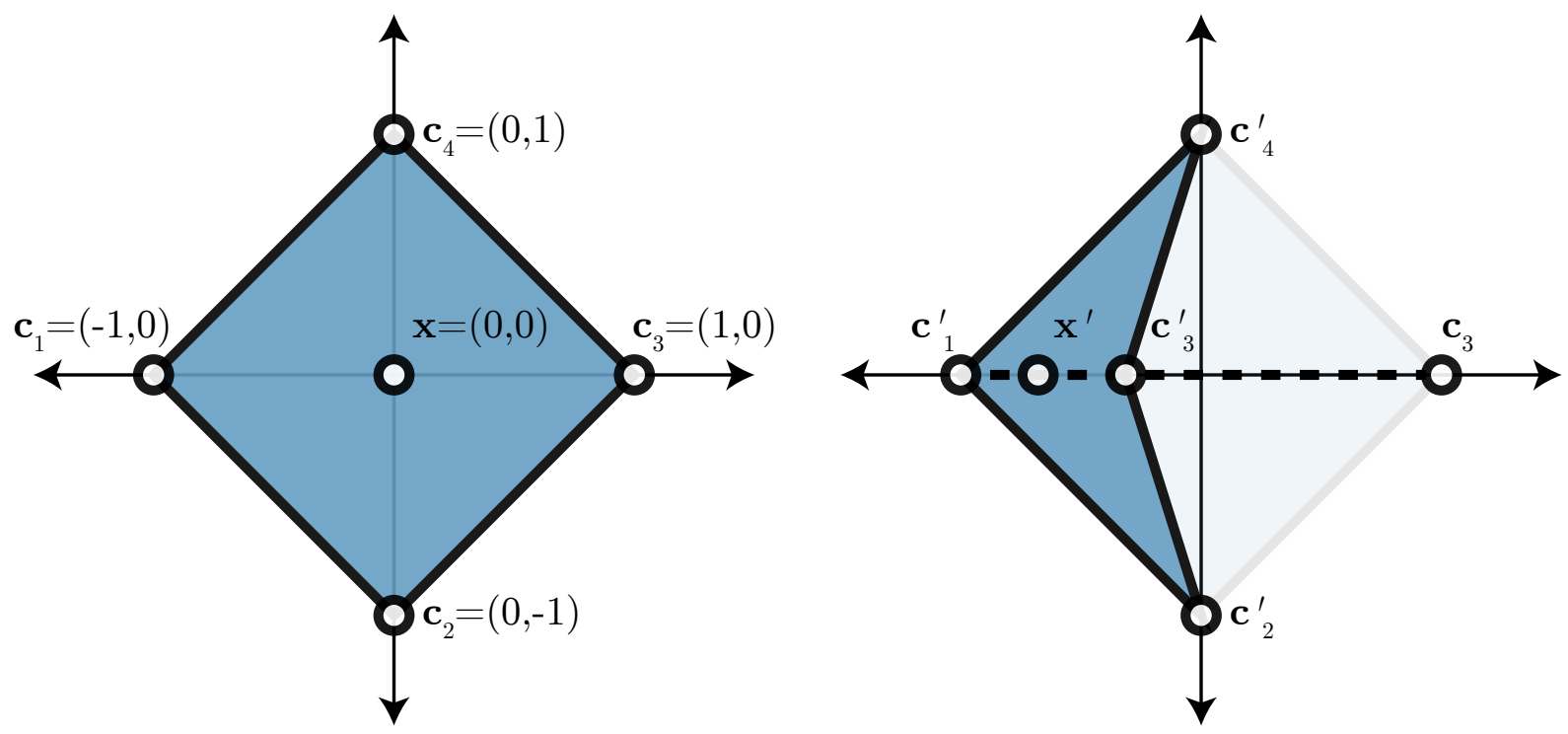

Figure 1: Consider the center of a square. Moving an arbitrary corner toward its opposite corner, we may show that a mapping produced by barycentric coordinates is either non-bijective or the coordinate corresponding to this corner is $1 / 2$. Because the choice of corner was arbitrary and there are four corners this contradicts the partition of unity property.

\section{Abstract}

Many recent works attempt to generalize barycentric coordinates to arbitrary polygons. I construct a counterexample proving that no such generalization will produce purely bijective mappings in the plane provided the coordinates meet the Lagrange, reproduction, and partition of unity properties. The proof concerns generalized barycentric coordinates in a square, but trivially generalizes to arbitrary polygons with degree greater than three.

Keywords: generalized barycentric coordinates, planar mappings

Barycentric coordinates in a triangle boast a list of favorable properties making them useful in a number of important tasks across fields (e.g. scattered data interpolation). A recent resurgence of work attempts to generalized barycentric coordinates to arbitrary polygons. Typically these works maintain the basic properties of coordinates (Lagrange, reproduction, and partition of unity) and vary in the degree to which they support other properties: closed form expression [Floater 2003; Manson and Schaefer 2010], smoothness [Joshi et al. 2007], positivity [Lipman et al. 2007], and so on. However, triangular barycentric coordinates possess an elusive property, so far unobtained by generalizations: the ability to produce bijective mappings in the plane. When used to define a planar map, barycentric coordinates produce an affine mapping which is trivially bijective so long as the map does not degenerate (the original triangle should map to a non-degenerate triangle). In the rest of this document I show by counterexample how such bijective mappings are unobtainable by any arbitrary generalized barycentric coordinates in a square. The counterexample trivially extends to any polygon of degree greater than three.

Consider a square $S$ with corners $\left\{\mathbf{c}_{1}, \mathbf{c}_{2}, \mathbf{c}_{3}, \mathbf{c}_{4}\right\}$ at respective
Cartesian coordinates $\{(-1,0),(0,-1),(1,0),(0,1)\}$. Define a barycentric coordinates mapping inside this square as a map:

$$
\mathcal{M}(\mathbf{x}): S \rightarrow \mathbb{R}^{2}=\sum_{i=1}^{4} w_{i}(\mathbf{x}) \mathbf{c}_{i}^{\prime}
$$

where $\mathbf{c}_{i}^{\prime}$ are the new or deformed positions of $\mathbf{c}_{i}$ and $w_{i}$ are scalar barycentric coordinate functions defined for each point $\mathbf{x}$ in and on the square, obeying the following properties:

- Lagrange: $w_{i}\left(\mathbf{c}_{j}\right)=\delta_{i j}$ and thus $\mathcal{M}\left(\mathbf{c}_{i}\right)=\mathbf{c}_{i}^{\prime}$

- Reproduction: $\sum_{i=1}^{4} w_{i}(\mathbf{x}) \mathbf{c}_{i}=\mathbf{x}$

- Partition of unity: $\sum_{i=1}^{4} w_{i}(\mathbf{x})=1$

We call an arrangement of $\mathbf{c}_{i}^{\prime}$ non-degenerate if the quadrilateral $\left\{\mathbf{c}_{1}^{\prime}, \mathbf{c}_{2}^{\prime}, \mathbf{c}_{3}^{\prime}, \mathbf{c}_{4}^{\prime}\right\}$ remains simple.

Theorem: For any such functions $w_{i}$, there exists a non-degenerate arrangement of $\mathbf{c}_{i}^{\prime}$ such that the mapping is not injective. That is, there always exists some $\mathbf{x}$ and $\mathbf{y}$ such that $\mathbf{x} \neq \mathbf{y}$ but $\mathcal{M}(\mathbf{x})=$ $\mathcal{M}(\mathbf{y})$.

Sketch: The idea of the proof is that as we bring $\mathbf{c}_{3}^{\prime}$ toward $\mathbf{c}_{1}$, one of three things will happen:

1. $\mathcal{M}(\mathbf{0})$ will reach $\mathbf{c}_{1}$ before $\mathbf{c}_{3}^{\prime}$ does,

2. $\mathbf{c}_{3}^{\prime}$ will overrun $\mathcal{M}(\mathbf{0})$ before $\mathbf{c}_{3}^{\prime}$ reaches $\mathbf{c}_{1}$, or

3. $w_{3}(\mathbf{0})=1 / 2$, contradicting partition of unity. 
Proof: Applying the reproduction property, we may rewrite the mapping formula as:

$$
\mathcal{M}(\mathbf{x})=\sum_{i=1}^{4} w_{i}(\mathbf{x})\left(\mathbf{c}_{i}^{\prime}-\mathbf{c}_{i}+\mathbf{c}_{i}\right)=\mathbf{x}+\sum_{i=1}^{4} w_{i}(\mathbf{x})\left(\mathbf{c}_{i}^{\prime}-\mathbf{c}_{i}\right)
$$

Consider the mapping of the origin $\mathcal{M}(\mathbf{0})$ as we move $\mathbf{c}_{3}^{\prime}$ along the $x$-axis from $\mathbf{c}_{3}=(1,0)$ toward $\mathbf{c}_{1}=(-1,0)$ while keeping $\mathbf{c}_{i}^{\prime}=\mathbf{c}_{i}$ for $i \neq 3$. Until $\mathbf{c}_{3}^{\prime}$ finally reaches $\mathbf{c}_{1}$ then the mapping is non-degenerate. Immediately we may write

$$
\mathcal{M}(\mathbf{0})=\sum_{i=1}^{4} w_{i}(\mathbf{0})\left(\mathbf{c}_{i}^{\prime}-\mathbf{c}_{i}\right)=w_{3}(\mathbf{0})\left(\mathbf{c}_{3}^{\prime}-\mathbf{c}_{3}\right)
$$

By noticing that $\mathbf{c}_{3}=-\mathbf{c}_{1}$ we may write

$$
\mathcal{M}(\mathbf{0})=w_{3}(\mathbf{0})\left(\mathbf{c}_{3}^{\prime}+\mathbf{c}_{1}\right)
$$

The remainder of the proof will deal only with the $x$-coordinate of the relevant points. I will use an nonbold font to refer to the scalar $x$-coordinate, so that $c_{3}^{\prime}$ is the $x$-coordinate of $\mathbf{c}_{3}^{\prime}$.

Assume for any choice of $c_{3}^{\prime}$ between $c_{1}$ and $c_{3}$ we have an injective map. I now draw a contradiction for every possible weight $w_{3}(\mathbf{0})$.

Case 1: Consider the case when $w_{3}(\mathbf{0})>1 / 2$ and let $\mathcal{M}(\mathbf{0})=\mathbf{c}_{1}$ :

$$
w_{3}(\mathbf{0})\left(\mathbf{c}_{3}^{\prime}+\mathbf{c}_{1}\right)=\mathbf{c}_{1}
$$

Because $c_{3}^{\prime}+c_{1} \leq 0$ and $1 / 2<w_{3}(\mathbf{0})$ we may solve for $c_{3}^{\prime}$ :

$$
\begin{aligned}
\frac{1}{2}\left(c_{3}^{\prime}+c_{1}\right) & >w_{3}(\mathbf{0})\left(c_{3}^{\prime}+c_{1}\right)=c_{1} \\
c_{3}^{\prime}+c_{1} & >2 c_{1} \\
c_{3}^{\prime} & >c_{1}
\end{aligned}
$$

which means that $\mathcal{M}(\mathbf{0})=\mathbf{c}_{1}=\mathcal{M}\left(\mathbf{c}_{1}\right)$ before degeneracy. So if we want an injective mapping then $w_{3}(\mathbf{0}) \leq 1 / 2$.

Case 2: Consider $w_{3}(\mathbf{0})<1 / 2$, and let $\mathcal{M}(\mathbf{0})=\mathbf{c}_{3}^{\prime}$.

$$
w_{3}(\mathbf{0})\left(c_{3}^{\prime}+c_{1}\right)=c_{3}^{\prime}
$$

and now since $w_{3}(\mathbf{0})<1 / 2$ we solve again for $c_{3}^{\prime}$ :

$$
c_{1}<c_{3}^{\prime}
$$

So once again, $\mathcal{M}(\mathbf{0})=\mathbf{c}_{3}^{\prime}=\mathcal{M}\left(\mathbf{c}_{3}\right)$ before degeneracy. Together with Case 1, if we want an injective mapping then $w_{3}(\mathbf{0})=1 / 2$.

Case 3: We now know that $w_{3}(\mathbf{0})=1 / 2$, but by rotational symmetry of our problem we may repeat our logic above showing that $w_{i}(\mathbf{0})=$ $1 / 2$ for $i=1,2,4$. Thus $\sum_{i=1}^{4} w_{i}(\mathbf{0})=2$ which is a contradiction to the partition of unity property.

Extending this counter example to other polygons simply involves considering a map from the original polygon to the given square (e.g. by mapping four corners to the square's corners and the remaining corners to somewhere along the respective sides). Then one may follow the same logic above.

This counterexample shows that no real-valued generalized barycentric coordinates induce mappings that are always bijective. It would be interesting to consider whether so-called complex barycentric coordinates [Weber et al. 2009] could provide such mappings. These coordinates often relax the Lagrange property so it is not clear to what extent these may be considered "coordinates". Nonetheless, pursuit of an analogous counterexample is interesting and I hypothesize fruitful.

\section{Acknowledgements}

I am grateful to Kai Hormann for an illuminating discussion.

\section{References}

FloAter, M. S. 2003. Mean value coordinates. Computer-Aided Geometric Design 20, 1, 19-27.

Joshi, P., Meyer, M., DeRose, T., Green, B., And SAnocki, T. 2007. Harmonic coordinates for character articulation. ACM Trans. Graph. 26, 3, 71.

Lipman, Y., Kopf, J., Cohen-Or, D., And Levin, D. 2007. GPU-assisted positive mean value coordinates for mesh deformations. In Proc. SGP, 117-124.

MAnson, J., And Schaefer, S. 2010. Moving least squares coordinates. In Proc. SGP, 1517-1524.

Weber, O., Ben-Chen, M., And Gotsman, C. 2009. Complex barycentric coordinates with applications to planar shape deformation. Comput. Graph. Forum 28, 2, 587-597. 